\title{
VON NEUMANN AND LATTICE THEORY
}

\section{GARRETT BIRKHOFF}

1. Introduction. John von Neumann's brilliant mind blazed over lattice theory like a meteor, during a brief period centering around 1935-1937. With the aim of interesting him in lattices, I had called his attention, in 1933-1934, to the fact that the sublattice generated by three subspaces of Hilbert space (or any other vector space) contained 28 subspaces in general, to the analogy between dimension and measure, and to the characterization of projective geometries as irreducible, finite-dimensional, complemented modular lattices.

As soon as the relevance of lattices to linear manifolds in Hilbert space was pointed out, he began to consider how he could use lattices to classify the factors of operator-algebras. One can get some impression of the initial impact of lattice concepts on his thinking about this classification problem by reading the introduction of [62],1 in which a systematic lattice-theoretic classification of the different possibilities was initiated. In particular, he saw that factors of Type $\mathrm{II}_{1}$ gave rise to continuous-dimensional modular subspace lattices.

However, von Neumann was not content with considering lattice theory from the point of view of such applications alone. With his keen sense for axiomatics, he quickly also made a series of fundamental contributions to pure lattice theory. The primary aim of the following paragraphs is to sketch these contributions. ${ }^{2}$

2. Continuous geometries. Von Neumann's major contributions to lattice theory centered around his concepts of a "continuous geometry" and of a "regular ring." Brief announcements of his ideas appeared in the Proceedings of the National Academy of Sciences $[66 ; 67 ; 68 ; 73 ; 74]$. Full expositions were published only as mimeographed notes (Continuous geometry, Institute for Advanced Study Lecture Notes, Spring, 1936; Continuous geometry, 1936-1937, Lecture Notes, Edwards Bros., 1937). See also [65] and [76].

One can construct a continuous-dimensional projective geometry $C G(F)$ over an arbitrary division ring $F$, as follows. For any $n$, the

Received by the editors January $23,1958$.

1 References in brackets refer to the Bibliography of John von Neumann, 19031957 which appears on page 1 of this volume.

2 Another survey of von Neumann's work on continuous geometry, by Israel Halperin, will appear in a Hungarian journal. I have borrowed freely from this source, and am also indebted to I. Kaplansky and S. Ulam for helpful comments. 
subspaces of the $2^{n}$-dimensional vector space over $F$ form a $\left(2^{n}-1\right)$ dimensional projective geometry $P G\left(F, 2^{n}-1\right)$. Moreover

$$
P G\left(F, 2^{n}-1\right)
$$

can be embedded isomorphically in $P G\left(F, 2^{n+1}-1\right)$, with preservation of normalized dimension $\delta[x]=(d[x]-d[0]) /(d[I]-d[0])$, where $I$ is the whole space. Further, both are metric spaces under the distance function

$$
|x-y|=\delta[x \cup y]-\delta[x \cap y],
$$

and the operations $\cap, \cup$ are uniformly continuous (with Lipschitz modulus one) in this topology. Hence, the metric completion of these spaces is itself a complemented modular lattice, which is irreducible and has a "dimension" function $\delta[x]$ ranging continuously between $0=\delta[0]$ and $1=\delta[I]$. This completion is the continuous geometry $C G(F)$; factors of Type $\mathrm{II}_{1}$ in Hilbert spaces have invariant subspace lattices isomorphic to such continuous geometries. Curiously, the real and quaternion continuous geometries are isomorphic.

Much more difficult is the converse problem, of characterizing the class of $C G(F)$ in abstract lattice-theoretic terms. Von Neumann's solution of this problem required him to develop many basic new ideas.

First, he gave a very successful abstract treatment of dimension in complete complemented modular topological lattices. Dimension is determined, up to a positive linear transformation, by the following two properties. It is conserved by perspective mappings ("perspectivities") and ordered by inclusion. By an elaborate and ingenious argument, the classes of perspective elements can be proved (in the irreducible case) to be isomorphic to the real numbers $0 \leqq d \leqq 1$ (or to the fractions $0,1 / n, 2 / n, \cdots, 1$ for some $n$ ). The deepest part of the proof concerns the equivalence of perspectivity with "projectivity by decomposition"-of which a corollary is the transitivity of perspectivity.

3. Regular rings. If $n$ is any positive integer exceeding three, then every $n$-dimensional "abstract" projective geometry is isomorphic with the subspace-lattice of an $n$-dimensional vector space $V_{n}(F)$ over a (unique) corresponding division ring $F$. This is also true of Desarguesian plane projective geometries. Von Neumann's partial extension of this fundamental result to the continuous-dimensional case is a truly remarkable feat of logical analysis and ingenuity. ${ }^{3}$

${ }^{8}$ For an analog of the plane Desargues case, see K. D. Fryer and I. Halperin, Acta Szeged vol. 20 (1956) pp. 203-249. 
He first gave the problem of "coordinatization" (i.e., of introducing coordinates) a radically new turn. He replaced the vector space $V_{n}(F)$ by the ring of "projectivities" generated by "perspectivities." In the finite-dimensional case, this ring is a simple full matrix algebra $M_{n}(F)$ $=R$ (modulo scalars; we shall not enter into this qualification). $P G(F, n)$ is then isomorphic with the lattice $R(R)$ of right-ideals of $R$.

In operator theory, direct sums of projective geometries occur as well; their projectivities correspond to general semi-simple algebras. To generalize this concept to the continuous-dimensional case, von Neumann coined a new definition: that of regular ring.

A regular ring is a ring in which, for every $a$, an element $x$ exists such that $a x a=a$. Regular rings having a finite basis over a division ring $F$ are just the "semi-simple" rings of the classical Wedderburn theory and so direct sums of simple rings. Any finite-dimensional complemented modular lattice is the direct union of a Boolean algebra, with (exceptional) non-Desarguesian geometries, with a direct union of Desarguesian geometries. The latter can be represented as the lattice $R(R)$ of right-ideals of the (regular or semi-simple) direct sum of the full matrix algebras coordinatizing the Desarguesian geometries in question.

In the general case, von Neumann proved the following basic representation theorem. Any complemented modular lattice $L$ having a "basis" of $n \geqq 4$ pairwise perspective elements, is isomorphic with the lattice $R(R)$ of all principal right-ideals of a suitable regular ring $R$. This conclusion is the culmination of 140 pages of brilliant and incisive algebra involving entirely novel axioms. Anyone wishing to get an unforgettable impression of the razor edge of von Neumann's mind, need merely try to pursue this chain of exact reasoning for himself-realizing that often five pages of it were written down before breakfast, seated at a living room writing-table in a bathrobe.

4. Related results. Von Neumann's fertile mind developed new technical ideas as needed, in proving the results described above. Many of these concern distributivity: the properties of elements distributive with arbitrary pairs in a complemented modular lattice, the distributivity of "independent" elements, and infinite distributivity.

The first of these had been analyzed by Ore, who defined the "neutral" elements of a modular lattice as those distributive with any other pair. Von Neumann quickly saw that the direct decompositions of a complemented modular lattice corresponded to its "neutral" elements. The set of these is a Boolean algebra, which he called the 
"center." He proved the striking result that the "center" of a complemented modular lattice consists of the elements having unique complements.

He was also the first to show that, in any Boolean algebra, the join and meet operations are necessarily infinitely distributive, and to recognize this distributivity as equivalent to continuity. ${ }^{4}$

Von Neumann also developed much of the theory of valuations in lattices. One can describe dimension, measure and probability functions as all "positive valuations" on appropriate lattices. Von Neumann's background in measure theory $([14 ; 28]$, etc.) made it easy for him to analyze the properties of such valuations. Thus, he showed that the "irreducible" factors of a complemented modular lattice correspond to its different linearly independent valuations. Again, he shared in developing the basic general theory of metric lattices, including the relation between metric convergence and star-convergence. Unfortunately, his speculations on the role of $d[x]$ in quantum mechanics, as an "a priori thermodynamic weight of states" [65, p. 833], never crystallized into a simple formal theory. ${ }^{5}$

Von Neumann's brilliance was not confined to mathematics. $\mathrm{He}$ coined the humorous name "pointless geometries" for continuousdimensional projective geometries. He did not publicize the name, leaving to others the pleasure of rediscovering the pun. He also chose a sequence of appropriate words to describe the computing machine he developed at the Institute for Advanced Study, whose initials spelled MANIAC. His friends dissuaded him from adopting the name officially; it is now privately called the JOHNIAC.

5. Other contributions. Though von Neumann's interest in lattice theory centered around possible applications to operator theory (and hence to the then new quantum mechanics), his scientific curiosity was also aroused by various other aspects of the subject. The satisfaction of this curiosity led to further results.

In fact, the lattice of closed subspaces of Hilbert space "reducing" (left invariant by) an operator does not necessarily satisfy the modular law,

$$
a \cap(b \cup c)=(a \cap b) \cup c \quad \text { if } a \geqq c .
$$

4 This result has recently been extended to orthocomplemented modular lattices by I. Kaplansky, Ann. of Math. vol. 61 (1955) pp. 524-541.

- His Fourth Colloquium Lecture (1937) was on Transition probabilities and quantum logics, but unpublished. The Institute for Advanced Study issued $17 \mathrm{pp}$. of mimeographed notes on Quantum logics in 1937. 
However, the subspaces reducing a self-adjoint operator form an ortho-complemented lattice-i.e., one with an intrinsic complementation satisfying

$$
\left(a^{\prime}\right)^{\prime}=a, \quad(a \cup b)^{\prime}=a^{\prime} \cap b^{\prime}, \quad(a \cap b)^{\prime}=a^{\prime} \cup b^{\prime},
$$

which is also weakly modular in the sense that

$$
a \cap\left(a^{\prime} \cup c\right)=\left(a \cap a^{\prime}\right) \cup c \quad \text { if } a \geqq c .
$$

These facts were well-known to von Neumann.

Since all these laws are satisfied in the finite-dimensional case, the principle of the persistence of formal laws suggests that the formal logic of quantum mechanics should conform to the algebraic laws of an orthocomplemented modular lattice. (The assumption $\left(a^{\prime}\right)^{\prime}=a$ is equivalent to the "tertium non datus" principle excluded in Brouwerian logic.) This idea was developed in a joint paper [65], where it was shown (this result was entirely von Neumann's) that $P G_{n}(F)$ admits such an "orthocomplementation" if and only if $F$ admits an involutory antiautomorphism. ${ }^{6}$

One aspect of the non-commutativity of the "quantum logic" is the failure of the distributive law. In general, one has only the semidistributive laws

$$
a \cap(b \cup c) \geqq(a \cap b) \cup(a \cap c) \text { and } a \cup(b \cap c) \leqq(a \cup b) \cap(a \cup c)
$$

which hold in an arbitrary lattice. Von Neumann quickly recognized these as special cases of the Minimax Inequality

$$
\bigwedge_{i=1}^{m} \bigvee_{j=1}^{n} a_{i j} \geqq \bigvee_{j=1}^{n} \bigwedge_{i=1}^{m} a_{i j}
$$

He also recognized that a central feature of the theory of games is provided by the fact ${ }^{7}$ that this inequality is replaced by equality

$$
\begin{aligned}
\underset{\boldsymbol{p}}{\operatorname{Min}} \operatorname{Max}_{\boldsymbol{q}} f(\boldsymbol{p}, \boldsymbol{q}) & =\underset{\boldsymbol{q}}{\operatorname{Max}} \operatorname{Min}_{\boldsymbol{p}} f(\boldsymbol{p}, \boldsymbol{q}) \\
& =-\left[\underset{\boldsymbol{q}}{\operatorname{Min}} \operatorname{Max}_{\boldsymbol{p}} f(\boldsymbol{p}, \boldsymbol{q})\right],
\end{aligned}
$$

for bilinear forms

$$
f(\boldsymbol{p}, \boldsymbol{q})=\sum p_{i} a_{i j} q_{j},
$$

$p$ and $q$ being variable probability vectors.

It is most remarkable that the preceding results were all obtained

- A similar result has been established for continuous geometries by F. Maeda, Journal of Science of the Hiroshima University vol. 14 (1950) pp. 93-96.

7 This is due to E. Borel, C. R. Acad. Sci. Paris (1927) pp. 52-55. 
in the four-year period 1934-1937, and mostly during two years of intense activity. Though his interest in lattice theory continued, and expressed itself in technical letters to other mathematicians, the subject was peripheral rather than central in his thoughts. (His paper [76] with Halperin was actually written and presented in 1936.)

In 1940 , he returned briefly to the subject, in preparing lectures for a seminar at the Institute for Advanced Study which we conducted jointly. Here, he developed the theory of $\sigma$-complete lattice-ordered rings with strong unit. He never wrote up this work for publication; it was closely related to work of M. H. Stone (Proc. Nat. Acad. Sci. U.S.A. vol. 26 (1940) pp. 280-283) done about the same time. Von Neumann's results were more general, because he did not assume the possibility of multiplication by real scalars.

Another characteristic late contribution is his part in the so-called Carathéodory-Halmos-von Neumann theorem [83], which displays the algebra of measurable subsets on $[0,1]$ modulo sets of measure zero as a universal separable Boolean algebra with positive valuation. However, a closer study of this highly technical paper reveals this as a very incidental result indeed. The main concern is with a much more difficult question: which Boolean algebras correspond to measurable sets under point-isomorphisms?

By this time, von Neumann was primarily preoccupied with wartime problems, and had developed an interest in computing and computing machines. Though his book with Morgenstern on the theory of games reflects continued interest in lattices [90, esp. pp. 62-66], he never really worked on them seriously after 1942 .

6. Extensions of his work. It is usually difficult to sharpen von Neumann's results. With small concern for expository simplifications or intuitive motivations, he characteristically went straight to the heart of problems, and had an uncanny ability to check all the essentially different possibilities, individually and in combination. This ability gives most of his work an objective finality, and makes later workers begin by trying to simplify von Neumann's arguments, or to apply similar techniques to related problems.

Nevertheless, since von Neumann's initial lightning attack, there has been significant progress on some of the problems described above. Thus, the theory of continuous geometries has been clarified and extended over the past twenty years, especially by Halperin, Maeda, Gorn, and Iwamura. (Since this work has been conveniently indexed in Mathematical Reviews under the heading "continuous geometries," it is unnecessary to refer to individual papers.) In this connection, one should also mention Loomis' recent analysis of di- 
mension functions in weakly modular, orthocomplemented lattices ${ }^{8}$ -an analysis which simplifies part of the discussion of [62].

Far less has been done to advance the theory of regular rings; this may be due to the formidable technical difficulties of the theory. It has been proved that a ring is regular if and only if $I \cap J=I J$ for a right-ideal $I$ and left-ideal $J$; also, if and only if it has (homological) weak dimension zero. ${ }^{9}$ Various other more special results have also been proved ${ }^{10}$-e.g., Brown and $\mathrm{McCoy}$ have replaced von Neumann's rather involved proof of the fact that the $n \times n$ matrix ring over any regular ring is regular, by a much simpler proof. Recently, much more work has been done on the related class of "Baer rings," or rings in which every annihilator is generated by an idempotent.

Again, the theory of metric lattices has been advanced by Smiley and Wilcox, Maeda, and Ky Fan. ${ }^{11}$ Some questions about valuation in Boolean algebras, touched on by von Neumann in his work on dimension functions, have been resolved by Halmos and Maharam. As this work belongs mainly to measure theory, it will not be discussed here. Also, von Neumann's unpublished idea on latticeordered groups have been partially extended by R. S. Pierce and myself to rings. ${ }^{12}$

Von Neumann's (and my) ideas about the logic of quantum mechanics have been carefully analyzed philosophically by Mme. Destouches-Favrier in two books. ${ }^{13}$ She has compared our views with those of de Broglie, J. Destouches, Reichenbach, and others. However, I know of no application of lattice-theoretic formulas to specific physical problems.

One wonders what would have been the effect on lattice theory, if von Neumann's intense two-year preoccupation with lattice theory had continued for twenty years!

HARVARD UNIVERSITY

8 Memoirs of the American Mathematical Society, no. 10, 1955.

- For these results, see M. Harada, Journal of the Institute of Polytechnics. Osaka City University vol. 7 (1956); M. Auslander, Proc. Amer. Math. Soc. vol. 8 (1957) pp. 658-664.

${ }^{10}$ For these results, see N. H. McCoy and Brown, Duke Math. J. vol. 13 (1946) pp. 9-20; F. Maeda, Journal of Science of Hiroshima University vol. 8 (1939) pp. 145167 and vol. 9 (1939) pp. 73-84. I am indebted to Irving Kaplansky for these references.

${ }^{11}$ References are given on p. 80 of the author's Lattice theory, Amer. Math. Soc. Colloquium Publications, no. 25, rev. ed., 1948.

12 Anais Acad. Bras. Ciencias vol. 28 (1956) especially p. 67.

${ }^{13} \mathrm{La}$ structure des theories physiques, and Determinisme et indeterminisme, Presses Universitaires de France, 1951, 1955. 